\section{CHEMOTHERAPEUTIC PITFALLS IN THE TREATMENT OF TUBERCULOSIS} BY

GRAHAM POOLE, M.B., M.R.C.P. Assistant Chest Physician

AND

PETER STRADLING, M.D., M.R.C.P. Chest Physician and Lecturer

From the Hammersmith Chest Clinic and the Postgraduate Medical School, Hammersmith Hospital, London

"I find the medicine worse than the malady." Francis Beaumont and John Fletcher. The Lovers' Progress, III, ii.

One of the greatest disasters that can befall a tuberculous patient is that his organisms should become resistant to two or more of the standard antituberculosis drugs, thereby denying him the most effective chemotherapy. Thus for those treating tuberculosis, avoidance of drug resistance has become a central theme of their work. Two known facts are of particular relevance to this problem. Anti-tuberculosis drugs employed singly, even in adequate dosage, lead to resistance when given for any length of time (Medical Research Council, 1950, 1952). The classical trials carried out by the Medical Research Council (1950, 1952, 1955) have also shown that certain dual-drug dosage schedules are fully acceptable as regards both efficacy and avoidance of resistance, provided the organisms are fully sensitive before the start of treatment. What is more, if such treatment is maintained for a sufficient period, up to $100 \%$ of sputum conversions can be expected (Middlebrook, 1956 ; Crofton, 1957). Thus it would seem that little difficulty should arise in the treatment of tuberculosis and that the disease could be managed easily by practitioners following rules based upon this knowledge. But one of the most difficult and not uncommon tasks facing the chest physician to-day is managing the patient who is found to harbour resistant organisms and who therefore does not respond to standard therapy. Misapplication of basic rules must thus be occurring.

In an effort to establish this, and more fully to understand the various causes of the emergence of resistant organisms, the clinical records of patients harbouring such organisms have been reviewed. To enable any conclusions to be drawn from these records it was necessary to assume certain basic "correct" methods of drug treatment with which comparison of actual treatments could be made. The M.R.C. schemes mentioned above were accepted for this purpose, and in this article other schedules are termed "faulty."

Another factor, already suspected as a cause of drug resistance, must be recognized when drawing conclusions from such records: the possibility that patients may not be consuming their orally prescribed medicaments. Thus if this occurs during the established administration of one drug the patient will receive this drug alone and strains of Mycobacterium tuberculosis resistant to it are likely to appear. It is reasonable to infer, conversely, that if correct dual therapy has been prescribed for a patient with drug-sensitive organisms and if resistance develops to a drug known to have been taken, then it is almost certain that the other drug was omitted. This reasoning has been followed in the present paper.
Finally, unless there is knowledge of the pretreatment drug-sensitivity pattern of the organisms there must be some uncertainty about any conclusions drawn. Particularly does this become significant when it is realized that some $5 \%$ of newly diagnosed untreated sufferers harbour resistant bacilli, presumably contracted from other maltreated patients (Fox et al., 1957).

\section{Method}

With the foregoing points in view a careful examination was made of the full clinical records, still held at the Hammersmith Chest Clinic, of 32 patients with initially sensitive organisms and with resistance reported to one or more drugs. This group is selected in that, of a total of 51 patients with resistant organisms. 19 had been transferred elsewhere and were not available.

Most of the drug-sensitivity tests were carried out on Löwenstein-Jensen media slopes by the methods used at the Tuberculosis Chemotherapy Centre, Madras (1959). It is the practice in the case of streptomycin and sodium para-aminosalicylate (P.A.S.) to report results as resistance ratios (R.R.) because of variations in behaviour of the standard strain used for comparison $(\mathrm{H} 37 \mathrm{rV})$. Growth in relation to concen trations of isoniazid is not so varied, and results in terms of the absolute drug concentrations have been used. An organism is reported sensitive (S) to streptomycin or P.A.S. if the R.R. is below 4 and to isoniazid if there are less than 20 colonies growing on media containing $0.2 \mu \mathrm{g} . / \mathrm{ml}$. of isoniazid. Doubt is expressed $( \pm)$ with an R.R. of 4 or if 20 or more colonies grow on $0.2 \mu \mathrm{g} . / \mathrm{ml}$. of isoniazid but not on higher concentrations. Definite resistance is reported (+) with an R.R. of 8 or more, or significant growth on $1 \mu \mathrm{g} . / \mathrm{ml}$. In this study R.R.s of 32 or more and significant growth on $5 \mu \mathrm{g}$. $/ \mathrm{ml}$. or more are taken to mean a high degree of resistance $(++)$.

The courses of treatment recorded had been prescribed at a variety of chest clinics, hospitals, and sanatoria, including Hammersmith. The exact dosages and duration of administration of all chemotherapeutic agents given and the serial drug sensitivities were exactly tabulated in their time relationships. This tabulated information was transferred to time charts, enabling the emergence of drug-resistant organisms to be readily seen in relation to previous drug schedules. To illustrate this method four examples are given (Figs. 1-4).

Any factors bearing on the probable degree of co-operation over drug-taking shown by the patients were all recorded ; in particular, admissions of failure, their known co-operation in other respects, and the reports of urine-testing for P.A.S. In this way deductions drawn from therapy and resistance records were often reinforced.

Finally, the extent of initial disease, progress, and present clinical status of the patient were all recorded While no definite conclusions can be drawn from this latter information in a selected group some useful pointers to the significance of drug-resistance were hoped for.

\section{Results}

Assessment of the information showed that one main cause usually produced the first resistance to one drug (initial resistance). Because the drug to which the first resistance had developed was often persisted with but 
TABLE I.-Resistances Tabulated According to Type and Drug to Which They Developed

\begin{tabular}{|c|c|c|c|c|c|}
\hline & & \multicolumn{3}{|c|}{ Drug to which Resistance Developed } & \multirow{2}{*}{ Total } \\
\hline & & Streptomycin & Isoniazid & P.A.S. & \\
\hline $\begin{array}{l}\text { Initial resistance } \\
\text { Subsequent ," }\end{array}$ & & $\begin{array}{r}18 \\
3\end{array}$ & $\begin{array}{r}26 \\
3\end{array}$ & $\begin{array}{l}3 \\
6\end{array}$ & $\begin{array}{l}47 \\
12\end{array}$ \\
\hline Total ... & . & 21 & 29 & 9 & 59 \\
\hline
\end{tabular}

TABLE II.-Initial Resistances Tabulated According to Cause and

\begin{tabular}{|c|c|c|c|c|}
\hline \multirow{2}{*}{ Causes of Resistance } & \multicolumn{3}{|c|}{ Drugs to which Resistance Developed } & \multirow{2}{*}{ Total } \\
\hline & Streptomycin & Isoniazid & P.A.S. & \\
\hline 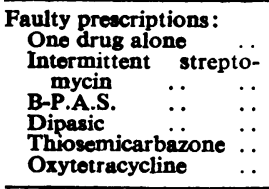 & $\begin{array}{l}\bar{z} \\
\frac{1}{1}\end{array}$ & $\begin{array}{r}6 \\
3 \\
2 \\
1 \\
-\end{array}$ & $\frac{-}{-}$ & $\begin{array}{l}3 \\
2 \\
1 \\
1 \\
1\end{array}$ \\
\hline Total $\ldots$ & 1 & 12 & 1 & 14 \\
\hline $\begin{array}{l}\text { Failure to take adequate } \\
\text { P.A.S. (including } 6 \text { pre- } \\
\text { ecriptions of combined } \\
\text { cachets) }\end{array}$ & 17 & 14 & 2 & 33 \\
\hline Total .. & 18 & 26 & 3 & 47 \\
\hline
\end{tabular}

gave no preventive cover, resistance to other drugs followed (subsequent resistance). A total of 59 examples of drug-resistance developed in 32 patients: 29 under treatment at home, 22 in sanatorium, and 8 in hospital. Of these, 47 were initial resistances and 12 subsequent (Table I). No example of resistance remained unexplained by the method adopted.

The main causes of initial resistance (Table II) were twofold. Firstly, it was deduced that patients failed to take adequate amounts of the prescribed oral P.A.S. in 33 cases $(70 \%)$. In $27(58 \%)$ the main cause of resistance appeared to be failure to take P.A.S. when prescribed in proper doses but separate from a second drug (Figs. 1 and 2). In 6 (13\%) cases resistance developed in spite of the prescriptions of P.A.S. plus isoniazid, in correct dosage in single cachets containing both drugs (Fig. 3): in one of these the patient admitted to irregular low consumption and in two others the urine tests for P.A.S. were sometimes negative.

Secondly, six varieties of faulty prescription occurred in 14 cases $(30 \%)$, the commonest being isoniazid given

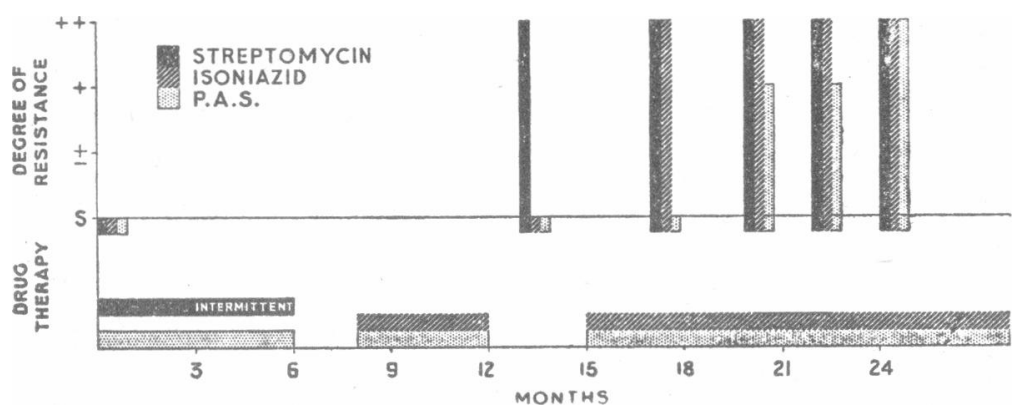

Fia. 1.-Case 3. During the first and second cuurses of chemotherapy this patient took (by admission) inadequate amounts of P.A.S., leading first to streptomycin resistance and then to isoniazid resistance. Finally, P.A.S. resistance developed after prolonged consumption of inadequate P.A.S. taken with isoniazid to which resistance had already appeared. alone. It is suggested that in two cases the explanation lies in the inherent deficiency of calcium benzamidosalicylate (B-P.A.S.). This is likely because these patients, who remain fully sensitive for long periods of treatment with P.A.S. plus one other drug, thus revealing that they took P.A.S. well, yielded resistant organisms

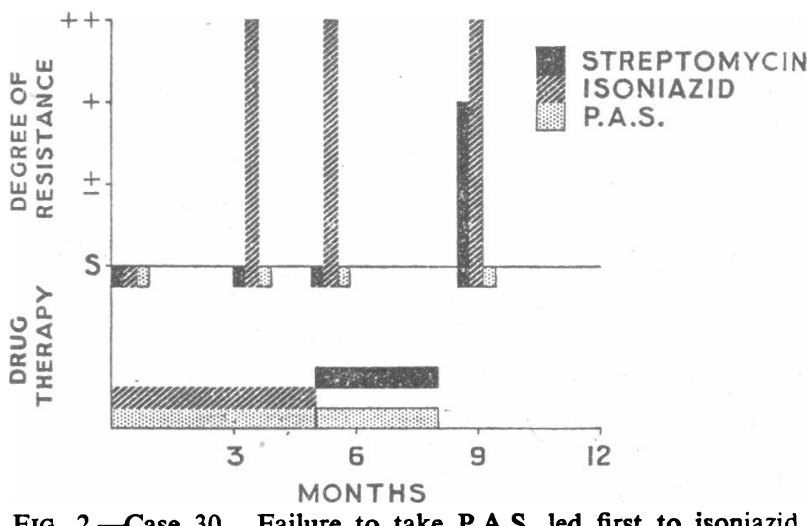

Fig. 2.-Case 30. Failure to take P.A.S. led first to isoniazid resistance and then to streptomycin resistance.

for the first time after changing to B-P.A.S. This is well seen in Fig. 4. The use of a thiosemicarbazone and that of oxytetracycline were both responsible for the development of resistance to an accompanying drug on one occasion each. Both streptomycin used intermittently and the proprietary preparation " dipasic' can also be incriminated.

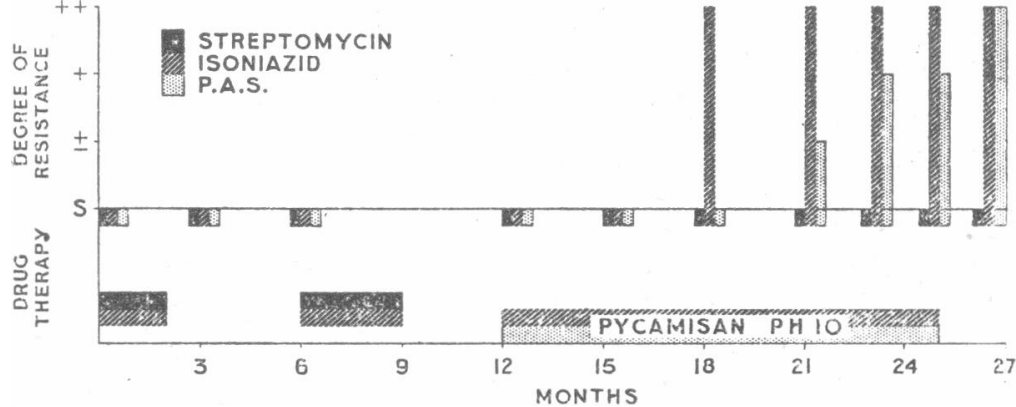

Fig. 3-Case 13. No resistance to streptomycin developed during five months of FIG. 3.- Case 13. No resistance to streptomycin developed during five months of treatment with this drug and isoniazid, indicating that the isoniazid was taken.
Subsequently, while taking inadequate amounts of "pycamisan," first isoniazid and then P.A.S. resistance followed.

The patients were classified according to extent of disease: 5 slight, 14 moderate, and 13 advanced-N.T.A. (1950) Classification Types 1, 2, and 3. Large cavities were present in six moderate and five advanced cases. With such small numbers, and the original population at risk unknown, it has been impossible to draw any definite conclusions relating this to the emergence of drug-resistant bacterial strains. Suffice it to say that the reasons for the resistance were equally readily found in all three groups and there was no evidence that it developed more quickly in the more advanced cases.

Particularly instructive is an analysis of the present position of the 32 patients. Only eight have quiescent disease and four of these needed major surgery to achieve this end. Of the remaining 24, 9 still have active disease and $15(47 \%)$ have died of their disease. To illustrate the significance of these figures 32 controls 
were obtained by means of a chronological survey of the clinic notification records. The first patient found matching for date of notification, age, sex, and extent of disease was accepted in each case provided chemotherapy had been given. Only two of these patients have died, and in neither case was death due to tuberculosis (one coronary thrombosis, one cerebral haemorrhage). The remaining 30 controls are alive and well. There is little doubt that the major causative factor

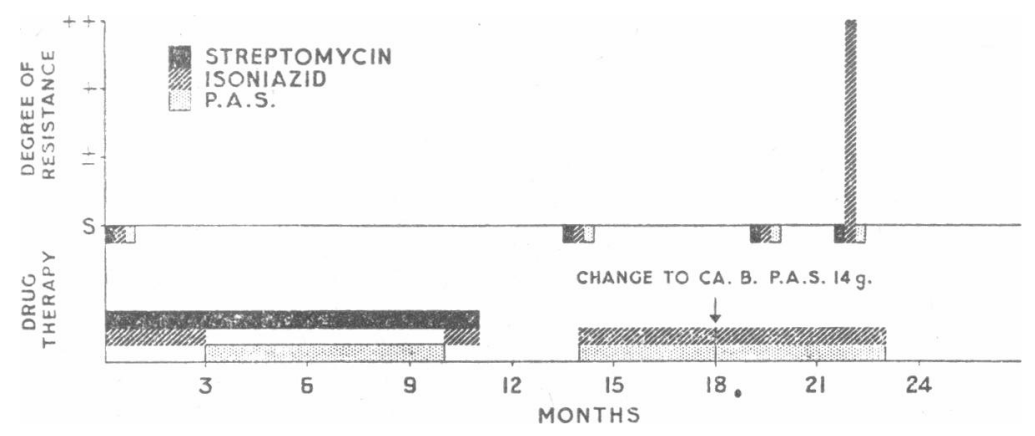

FIG. 4.-Case 5. In this case after seven months' treatment with streptomycin and P.A.S. and four months with isoniazid and P.A.S. the organism remained fully sensitive to all three drugs. This suggests that $\mathrm{P}$ A.S. was taken in effective doses. Resistance to isoniazid appeared only after substituting Ca B-P.A.S. (14 g.) for sodium P.A.S. (10 g.).

for this striking prognostic contrast is the loss of effective chemotherapy following the appearance of resistant organisms.

\section{Discussion}

Apart from prescribing one drug alone, the established cause of the development of resistance to one drug is that the other drug in dual therapy, or the other two in triple therapy, shall be taken in insufficient amount or ineffective form. Confusion may arise, however, over the world-wide divergence of opinion on the degree of bacterial resistance that is clinically significant (International Union against Tuberculosis, 1957). But the use of an insufficiently sensitive drug-resistance test, or the acceptance of too high a threshold for drug resistance, can lead to subsequent resistance to another drug used with that so tested and thus assumed effective. In this study, therefore, as described, bacterial growth on relatively low concentrations of drug in the test media have been accepted as indicating clinically significant resistance. The justification for this is further discussed by Crofton (International Union against Tuberculosis, 1957) and Mitchison and Selkon (1957).

In the light of these facts the results of this investigation suggest that, regardless of whether treatment takes place in the patients' homes or in an institution, or of the extent of the disease initially, the chief cause of the development of resistance lies in the failure to take adequate P.A.S. when administered separately. There was no evidence that patients failed to take separate tablets of isoniazid, and the very fact that six cases of isoniazid resistance developed when this drug was prescribed alone supports this. On the other hand, of the 59 resistances, only 9 developed to P.A.S. (Table I) though that drug was prescribed more often than any other in this series (P.A.S. 156 times; isoniazid 124 times ; streptomycin 114 times): resistance cannot develop to a drug that is not taken. A further point supporting the contention that P.A.S. is regularly rejected is related to the faulty prescription of intermittent streptomycin plus daily P.A.S. or isoniazid. If both drugs are taken in these circumstances P.A.S. or isoniazid resistance, respectively, would be expected In fact, in this series, nine patients received intermittent streptomycin plus daily P.A.S. and all yielded organisms resistant to streptomycin but not to P.A.S.

On the other hand, three prescriptions of intermittent streptomycin plus daily isoniazid led to the expected isoniazid resistance only. Direct evidence, by admission or from urine tests, was obtained that $6(19 \%)$ of the 32 patients had failed to take P.A.S., while 9 others $(28 \%)$ proved so uncooperative in other ways that co-operation in this respect was unlikely. Because the management of many of these cases antedated the common use of urine tests these figures almost certainly represent an understatement of the true position. It thus seems that there is more to the P.A.S. problem than the well-known occasional forgetfulness to consume a prescribed drug: to produce the results here presented there must be either a regular conscious omission of P.A.S. or doubt thrown upon the capacity of P.A.S. to prevent bacterial resistance to a second drug.

There is nothing new in the conclusion that patients often fail to take P.A.S. (Simpson, 1956 ; Dixon et al., 1957 ; Wynn-Williams and Arris, 1958; Fox, 1958 ; Rigby, 1958), and this is considered to be the most likely explanation for our findings. The results draw attention to the very serious outcome of this habit, so understandable when the drug is distasteful and no particular symptom is being relieved. Only in hospital can the patient be observed regularly when taking his medicine, so that P.A.S. is a very unsatisfactory drug for out-patient use. Even in hospital, however, the utmost vigilance is necessary, as the results show. It is now the practice in our wards at Hammersmith to watch every patient take every dose.

It is our experience that the true magnitude of this problem is still not widely recognized: even in the new edition of Recent Advances in Respiratory Tuberculosis (Heaf and Rusby, 1959) the failure to take P.A.S. when prescribed with isoniazid is only mentioned as a temptation, the disadvantage of which can be overcome by prescribing P.A.S. plus isoniazid in the same cachet. This investigation shows, however, that even cachets containing a combination of these drugs in accepted proportions cannot be relied upon to prevent resistance unless a fully effective dose is taken. This is most unfortunate in view of the widespread use of this type of combination under the misapprehension that the patient will either get effective treatment or, if he casts away the cachets, none (and so no resistance); regrettably, patients may take less than the prescribed dose, but reasonably regularly.

The findings also cast doubt upon the effectiveness of the usual dose of B-P.A.S. in preventing the emergence of resistant strains. Furthermore, we are not aware of any convincing evidence that B-P.A.S. is as effective as P.A.S. therapeutically. The in vitro experiments of Schönholzer et al. (1955) suggest that undissociated B-P.A.S. is a hundred times less effective than P.A.S. The serum levels of P.A.S. after taking B-P.A.S. are much lower than those after taking P.A.S., and the biological activity of the serum is only equivalent to the P.A.S. content (J. McD. Simpson, 1958, and Sheila Stewart, 1958, personal communications). Lewis (1958) 
has calculated that to achieve an absorption of P.A.S. equal to that after $20 \mathrm{~g}$. of sodium P.A.S. no less than 48 g. of B-P.A.S. daily would have to be prescribed.

Gibson and Nagley (1955) studied the use of B-P.A.S., but this was not a controlled trial. Many patients received streptomycin three times weekly for only two months $(25 \mathrm{~g}$.) so that the chance of the emergence of streptomycin resistance would not be high. From the information presented in their Table 2, relating to 27 patients remaining positive, one hesitates to draw any valid conclusions because so many had received previous chemotherapy and the majority no initial sensitivity tests. Only seven previously untreated patients received chemotherapy for more than three months and one of these for six. In spite of this inadequate period two examples of resistance to the other drug occurred in these seven cases. If therapy had continued further resistances might well have occurred: suboptimum doses of P.A.S. reduce and delay the emergence of resistance but will not prevent it. The paper of Phillips et al. (1957), sometimes quoted in support of the ability of B-P.A.S. to prevent drug resistance, gives no precise details of sputum states, drug combinations, or serial resistances. Lewis (1958) also presents evidence suggesting that B-P.A.S. is not effective in preventing the emergence of resistance to other drugs. In the absence of any dependable clinical trial giving clear evidence to the contrary, it is concluded that B-P.A.S. should never be used.

Failure to prescribe effective drug combinations or dosage schedules for newly diagnosed patients was a less frequent but equally important cause of resistance. The faulty schedules which apparently favoured emergence of resistant strains were seen to be varied in this series (Table II). Such prescriptions should now be rare and certainly ought not to be used in the future. Furthermore, to ensure safe treatment for the $5 \%$ of newly diagnosed patients who harbour resistant organisms to one of the three standard drugs, it should be an invariable practice to prescribe all three drugs daily to all new patients until drug sensitivities are known. It is implicit in this statement that strenuous efforts must be made to obtain organisms for culture before treatment is started. A little-realized point should be made here: while it is obviously somewhat safer (within the limitations already stated) to prescribe P.A.S. plus isoniazid together in single cachets on those occasions when it is the only possible dual combination, when prescribing the three drugs they should be given as separate entities. If the P.A.S. is not taken, then the isoniazid is likely. to remain coupled with the streptomycin.

With patients previously treated the problem can become acute: it may even be safest to await firm reports on present drug sensitivities before starting treatment, because in certain instances it will be safer to delay treatment than to risk the loss of yet another drug. Thus exceptionally strenuous efforts, using if necessary multiple laryngeal swabs and gastric and bronchial lavages, must be made to obtain a positive culture. The importance of taking a most careful and detailed history of previous drug treatment in such cases cannot be overemphasized: the exact drugs, their dosages, sequence, and duration of prescription, are vital. From such information it is usually possible to hazard correct guesses at the probable drug-resistance pattern of the organism. In particular it is suggested that whenever previous courses of treatment have involved the use of P.A.S., notably in separate form but also in cachets combined with isoniazid, then considerable suspicion should be entertained that a second drug used simultaneously may no longer be effective. Failure to realize this may well lead to multiple resistances and the loss of further drugs. The tragic possibilities are illustrated by the death rate in this series compared with that in the controls. To assist in the full appraisal of such cases a plea is made that hospitals, sanatoria, and clinics dealing with tuberculosis should make available as a first priority, on discharge or transfer, complete details of drug courses and sensitivities.

The supreme importance of choosing a drug combination likely to be suitable for a particular patient is thus obvious, but occasionally when faced with a difficult situation there is the temptation to use unproved remedies with apparently quite attractive properties This can lead to tragic results, particularly if accepted on a nation-wide scale, as, for example, in the case of B-P.A.S. and dipasic. These drugs should never be used-B-P.A.S. since its effectiveness is unproved. dipasic because its therapeutic and antibacterial effects are similar to those of isoniazid, and, when given alone as often recommended, isoniazid resistance develops (Walker et al., 1957 ; Allan et al., 1957 ; Williams and Prokopowicz, 1958).

The presence of organisms resistant to even one drug is a clear indication for treatment in hospital until the sputum is negative; the need in such a case to choose a suitable combination of drugs with great care is obvious. When resistance is multiple the judicious use of the more dangerous " second line" drugs, cycloserine, viomycin, pyrazinamide, and 1314 , certainly calls for full hospital resources.

The maintenance of drug therapy for at least 18 months is essential to ensure a good response and freedom from relapse (Stewart et al., 1956 ; Véran et al., 1957 ; Ross et al., 1958) ; commonly more is desirable. and anything less should be exceptional. This raises the problem of enforcing correct domiciliary treatment regimes after discharge from hospital. Obviously oral P.A.S. often proves unsatisfactory, and it is recommended that all patients with suitably sensitive organisms should receive daily streptomycin and isoniazid as a standard treatment, with the latter given under supervision at the time of the injection (Stradling, 1957: Dixon et al., 1957). Not only is this combination the best currently available (Medical Research Council. 1955), but it seems to be the only way at present to ensure adequate, regular dual-drug therapy. Effort put into making the necessary administrative arrangements (Stradling and Poole, 1958) will be amply repaid.

In circumstances where oral P.A.S. plus isoniazid is the only possible combination because of toxic reactions or an adverse resistance pattern, it is advised that frequent, irregular urine-testing for P.A.S. be resorted to. Every effort should be made to keep the true reason for the tests from the patient, and the test itself should detect only adequate doses of P.A.S. A suitable ferric chloride test is described by Dixon et al. (1957). If it is found that the patient is unreliable in any way a decision must be made either to stop treatment or to admit him to hospital: the risk of drug-resistant organisms emerging if out-patient therapy is continued, even if P.A.S. plus isoniazid is given in combined cachet form, is so great that if admission is declined it is safer 
to abandon treatment and risk a relapse. At least the organisms present under such circumstances will be fully sensitive and the patient responsive to re-treatment.

\section{Conclusion}

The apparent simplicity with which tuberculosis can now be treated is leading many physicians and practitioners to attempt the management of this disease, so long beyond their purview. It is thus opportune and salutary, particularly at this time, when moves are being made to return tuberculosis to the field of general medicine (Joint Tuberculosis Council, 1958), to point out the ease with which fundamental and dangerous errors can be made. All too often the tragic presence of multiple drug-resistant tubercle bacilli is iatrogenic and could have been avoided by the application of basic knowledge. At least two of the three proved drugs should always be used together in long-term treatment and in adequate daily dosage: $1 \mathrm{~g}$. of streptomycin, $200 \mathrm{mg}$. of isoniazid, and at least $10 \mathrm{~g}$. of P.A.S., though nearer $20 \mathrm{~g}$. of P.A.S. is advisable when given with streptomycin only.

The fact that much of the fault can be ascribed to the patients' failure to consume drugs cannot absolve the medical attendant from responsibility to his patient and-with an infectious disease-to the community. Knowing the position, he should seek means of applying, for an effective period, the forms of therapy which are, on the one hand, least open to criticism by the profession and, on the other, to abuse by the patient.

\section{Summary}

An analysis of 32 fully documented case histories yielded 59 examples of drug resistance in Mycobacterium tuberculosis.

In $33(70 \%)$ of 47 initially resistant examples the prescription of P.A.S. failed to prevent resistance to the other drug given. By far the most likely explanation of this is that P.A.S. was not consumed in adequate regular amounts. Evidence is also presented that P.A.S. plus isoniazid given together in single cachet form may also not be effective, perhaps for the same reason.

No evidence was found that isoniazid is similarly rejected by patients.

In 14 examples (30\%) faulty prescription of drugs was responsible for the resistance. This included the use of one drug alone, prescribing one daily and the other intermittently, the use of such unproved substances as B-P.A.S., dipasic, or a thiosemicarbazone, and also oxytetracycline given with streptomycin.

The importance is stressed (a) of using sensitive drugresistance tests and accepting a low threshold of resistance, $(b)$ of scrutinizing a patient's previous therapeutic history and obtaining sensitivities where possible before prescribing further anti-tuberculosis drugs in an effort to avoid resistances, (c) of avoiding the prescription of P.A.S. when its administration cannot be fully supervised, $(d)$ of never using drugs that have not shown themselves satisfactory in rigidly controlled clinical and bacteriological field trials (B-P.A.S. ; dipasic; thiosemicarbazones and their combinations such as "tebafan" and hinconstarch).

It is concluded that, until a more satisfactory oral agent can be found to accompany isoniazid, long-term daily streptomycin given with daily isoniazid is not only the most effective combination therapeutically but the only way of ensuring adequate treatment in spite of the patient's shortcomings.

The ease with which major therapeutic errors can be made with tragic consequences is fully stressed. This point is illustrated by the fact that 15 of the 32 patients in whom drug resistance occurred have since died of their disease: this is in stark contrast to the now usual results of correct long-term chemotherapy.

Our thanks are due to the staff of the Department of Bacteriology, Postgraduate Medical School of London, not only for the unsurpassed routine service provided but for constant advice and encouragement during our management of these difficult cases. Both Professor John Crofton, of Edinburgh, and Dr. D. A. Mitchison, of the Postgraduate Medical School, read the manuscript and offered much helpful constructive criticism.

ADDENDUM.-Since submitting this article for publication our conclusions that $(a)$ B-P.A.S. should not be employed, and $(b)$ even cachets containing isoniazid and sodium P.A.S. in accepted proportions cannot be relied upon to prevent drug resistance, have been strengthened respectively by Citron and Kuper (1959) and Simpson (1959). A further simple urine test for P.A.S., using " phenistix" (Ames Co., Inc.), has also been investigated by Luntz (1959): we can fully confirm its efficacy and greater simplicity.

\section{REFERENCES}

Allan, G. W., McNaught, W., and Lees, A. W. (1957). Lancet, 2. 609.

Citron, K. M., and Kuper, S. W. A. (1959). Tubercle (Lond.), 40,443 .

Crofton, J. W. (1957). Newc. med. J., 25, 45.

Dixon, W. M., Stradling, P., and Wootton, I. D. P. (1957). Lancet, 2, 871

Fox, W., Wiener, A., Mitchison, D. A., Selkon, J. B., and Sutherland, I. (1957). Tubercle (iond.), 38, 71 .

Fox, W. (1958). Ibid., 39, 269.

Gibson, M. O. J., and Nagley, M. M. (1955), Ibid., 36, 209. tory Tuberculosis, 5th ed. Churchill, London.

International Union Against Tuberculosis (1957). Bull. Un. int. Tuberc., 27, No. $3 / 4$

Joint Tuberculosis Council (1958). A Report on the Future Service for Tuberculosis and Diseases of the Chest.

Lewis, D. O. (1958). Tubercle (Lond.), 39, 247.

Luntz, G. R. W. N. (1959). Personal communication

Medical Research Council (1950). Brit. med. J., 2, 1073. (1952). Ibid., 2, 735 .

(1955). Ibid., 1, 435.

Middlebrook, G. (1956). Bull. Un. int. Tuberc., 26,179

Mitchison, D. A., and Selkon, J. B. (1957). Tubercle (Lond.), 38, 85.

National Tuberculosis Association (1950). Diagnostic Standards and Classification of Tuberculosis, 9th ed. New York

Phillips, S., Larkin, J. C., jun., Schlenker, F. S., and Ulrich, Eliz. W. (1957). Amer. Rev. Tuberc., 75, 667.

Rigby, J. P. V. (1958). Tubercle (Lond.), 39, 336.

Ross, J. D., Horne, N. W., Grant, I. W. B., and Crofton, J. W. (1958). Brit. med. J., 1, 237

Schönholzer, G., Lauener, H., and Hurni, H. (1955). Schweiz. med. Wschr. 85, 222 .

Simpson, J. McD. (1956). Tubercle (Lond.), 37, 333.

(1959). Ibid., 40, 453.

Stewart, Sheila M.; Turnbuil, F. W. A., and Macgregor, Agnes, R.'(1956). Ibid., 37, 388 .

Stradling, P. (1957). Proc. roy. Soc. Med., 50, 342.

- and Poole, G. W. (1958). Lancet, 2, 1066

Tuberculosis Chemotherapy Centre, Madras (1959). Bull. Wld Hlth Org., 21, 51.

Véran, P., Lucas, and Moigneteau, C. (1957). Rev. Tuberc. (Paris), 5 ser., 21, 39.

Walker, W. C., Hay, D., Stewart, Sheila M., and Crofton, J. W. (1957). Tubercle (Lond.), 38, 238.

Williams, H., and Prokopowicz, J. (1958). Ibid. 39, 201.

Wynn-Williams, N., and Arris, Margaret (1958). Ibid., 39, 138.

A list of the "Persons Employed in Druggists Shops in Edinburgh on the Sabbath Day" is given in a document, dated 1839, from the pharmacy of $\mathrm{H}$. C. Baildon and Son, Edinburgh, which, with others, has been presented to the Scottish History of Pharmacy Committee. The number employed was 273 , to serve a population of about 120,000 . (Pharmaceutical Society.) 